\title{
Escuchando a los niños: Significados sobre aprendizaje y participación como ejes centrales de los procesos de inclusión educativa en un estudio en escuelas públicas en Chile*
}

\author{
Listening to children: Meanings about learning and participation as central concepts of \\ inclusive education processes of a study in public schools in Chile

\begin{abstract}
Ouvindo as crianças: Significados sobre aprendizagem e participação como eixos centrais dos processos de inclusão em um estudo em escolas públicas no Chile
\end{abstract}

\author{
Natalia Albornoz, ${ }^{a}$ Natalia Silva, ${ }^{b}$ Mauricio López ${ }^{c}$ \\ ${ }^{a}$ Universidad de Chile, Estudiante del Magíster en Psicología Educacional, Santiago, Chile. \\ Teléfono: (56) 02 29787800. Correo electrónico: natalbmu@ug.uchile.cl \\ ${ }^{b}$ Universidad de Chile, Departamento de Psicología, Santiago, Chile. \\ Teléfono: (56) 02 29787824. Correo electrónico: natalia.silva@u.uchile.cl \\ 'Universidad de Chile, Departamento de Psicología, Santiago, Chile. \\ Teléfono: (56) 02 29787800. Correo electrónico: m.lopez@u.uchile.cl
}

\begin{abstract}
En el desarrollo de políticas que buscan atender a la diversidad de los estudiantes en Chile ha predominado una respuesta basada en la identificación de grupos específicos y en la implementación de estrategias de compensación de los supuestos déficits individuales, prestando poca o nula atención al derecho de los niños a participar en los asuntos que les afectan. Partiendo de esta premisa, en el presente estudio se indagó, mediante técnicas narrativo visuales, en los significados que los propios estudiantes construyen respecto a sus experiencias escolares de aprendizaje y participación, en el contexto de escuelas que están desarrollando proyectos de integración escolar. Los resultados sugieren que dichos significados enfatizan el vínculo interpersonal con profesores y compañeros, un clima de aprendizaje basado en la cooperación, el juego y la participación, así como la importancia del respeto, el trato justo y la no violencia. Estos hallazgos tienen implicancias claves para la orientación de las actuales políticas sobre inclusión educativa.
\end{abstract}

Palabras clave: inclusión educativa, significados, derecho a ser escuchado, aprendizaje y participación.

\section{ABSTRACT}

In developing policies that seek to address the diversity of students in Chile has dominated a response based on the identification of specific groups, and the implementation of strategies to compensate for the alleged individual deficits, paying little or no attention to the rights of children to participate in matters that affect them. Starting from this premise, the present study investigated, through visual narrative techniques, the meanings constructed by the students themselves about their school experiences of learning and participation, in the context of schools which are developing projects of integration. The results suggest that these meanings emphasize the interpersonal relationship with teachers and fellow students, a learning climate based on cooperation, play and participation as well as the importance of respect, fair treatment and non-violence. These findings have relevant implications to the orientation of current policies on inclusive education.

Key words: inclusive education, meanings, right to be heard, learning and participation.

\footnotetext{
Esta investigación se ha realizado dentro del proyecto número 11110141 subvencionado por Fondecyt-Chile.
} 


\section{RESUMO}

No desenvolvimento de políticas que visam atender a diversidade de estudantes no Chile, há domínio de uma resposta baseada na identificação de grupos específicos e na implementação de estratégias de compensação de supostos déficits individuais, dando pouca ou nenhuma atenção aos direitos das crianças de participar de assuntos que lhes dizem respeito. Partindo dessa premissa, investigaram-se, utilizando técnicas narrativas visuais, os significados construídos pelos próprios estudantes sobre suas experiências escolares de aprendizagem e participação, no contexto das escolas que estão desenvolvendo projetos de integração escolar. Resultados sugerem que tais significados enfatizam o relacionamento interpessoal com professores e companheiros, um clima de aprendizagem baseado na cooperação, o jogo e a participação, bem como a importância do respeito, tratamento justo e a não violência. Tais conclusões têm implicações chave para a orientação das políticas atuais sobre a inclusão educativa.

Palavras chave: inclusão educativa, significados, direito de ser ouvido, aprendizagem e participação.

\section{INTRODUCCIÓN}

En la investigación sobre inclusión educativa se aprecia un creciente interés por la perspectiva de los propios niños y jóvenes, coherente con el reconocimiento de los derechos del niño como parte de los fundamentos éticos y filosóficos de la educación de calidad (Blanco, 2006). En este sentido, en el último tiempo se ha prestado una atención considerable a la interpretación e implementación del derecho del niño a ser escuchado como uno de los principios fundamentales en que se basa la convención de los derechos del niño, reconociéndolos como protagonistas activos, con el derecho de participar en las decisiones que afectan sus vidas (Lansdown, 2005). Más específicamente, se ha argumentado que las perspectivas narrativas que buscan dar voz a jóvenes excluidos pueden iluminar temas pocos visibles para el mundo académico propio de los investigadores (Parrilla, 2009).

Por otra parte, en el desarrollo de políticas que buscan atender a la diversidad de los estudiantes en Chile ha predominado una respuesta basada en la identificación de grupos específicos y en la implementación de estrategias de compensación de los supuestos déficits individuales. Esta forma restringida de abordar la respuesta a la diversidad conlleva una concepción estática del desarrollo, que categoriza a los estudiantes en base a sus dificultades para aprender y otorga a estos escasa o nula participación en las acciones que les afectan (Infante, 2007). Persisten, por tanto, prácticas educativas basadas en la intervención de profesionales especialistas de modo remedial e individual con niños identificados como portadores de un problema (López et al., 2014).

De este modo, las políticas desarrolladas los últimos años para atender la diversidad constituyen un escenario poco propicio para al desarrollo de una perspectiva inclusiva de la escuela, donde se plantean numerosos desafíos. Estos desafíos pueden ser resumidos en la idea de la necesidad de un cambio cultural profundo en las políticas nacionales de inclusión, las prácticas pedagógicas en las escuelas y las concepciones de los actores educativos. Parte de este cambio cultural significa superar lo que Slee (2011) denomina el "sistema de racionalidad" de la integración, es decir, que si bien hay ajustes en el lenguaje que propone un enfoque más inclusivo de la educación, el modo de pensar y operar en la práctica sigue siendo el de integración.

En el marco de la investigación, entendemos la educación inclusiva como el proceso continuo de búsqueda de una educación de calidad para todos, respondiendo a la diversidad y a las diferentes necesidades, habilidades, características y expectativas de aprendizaje de los estudiantes y comunidades, eliminando todas las formas de discriminación (United 
Nations Educational, Scientific and Cultural Organization [UNESCO], 2008). De igual modo, puede entenderse como la tarea de identificar y superar las barreras que limitan la presencia, el aprendizaje y la participación de todos los niños y niñas, barreras que se encuentran principalmente en las culturas, políticas y prácticas escolares, y no en las características del niño (Booth \& Ainscow, 2000).

Los conceptos de aprendizaje y participación, por tanto, son fundamentales para comprender la perspectiva de inclusión educativa. En este contexto, "aprendizaje" se refiere a que todos los estudiantes progresen en sus capacidades y desarrollen su máximo potencial, mediante experiencias educativas amplias, relevantes y significativas para su vida, que no solo apuntan al rendimiento académico (Ainscow \& Miles, 2009). En este sentido, tal como ha sido propuesto en el Diseño universal de aprendizaje (CAST, 2008), para favorecer el aprendizaje de todos los estudiantes es necesario asegurar que el diseño de materiales y actividades curriculares considere múltiples medios de representación de los contenidos por parte del profesor, múltiples formas de expresión y comunicación de los contenidos por parte de los estudiantes y múltiples formas de motivación que respondan a diversos intereses de estos.

Como uno de los valores inclusivos, la participación significa estar y colaborar con otros, implicarse activamente en la toma de decisiones, reconocer y valorar una variedad de identidades, que todos sean aceptados por quienes son (Ainscow et al., 2006). La participación implica aprender junto con otros y colaborar en lecciones compartidas mediante la implicación activa con lo aprendido y enseñado, siendo reconocido y aceptado por ser quien es (Black-Hawkins et al., 2007). La participación involucra todos los aspectos de la vida escolar, requiere un aprendizaje activo y colaborativo de todos y está basada en relaciones de mutuo reconocimiento y aceptación. El foco en la participación aporta a la inclusión la noción de involucramiento activo, que implica: acceso (estar ahí), colaboración (aprender juntos) y diversidad (reconocimiento y aceptación).

Para abordar tanto aprendizaje como participación resulta relevante prestar atención al proceso de desarrollo de niños y niñas y a las condiciones en que este se da. Al respecto, la perspectiva sociocultural aporta una comprensión del desarrollo más coherente con un enfoque de derechos (Lansdown, 2005). El desarrollo se concibe desde esta perspectiva como un proceso de transformación del niño y su entorno a través de la apropiación de herramientas que la cultura ofrece y de la participación en los problemas y desafíos de la vida cotidiana (Rogoff, 1997). Por lo tanto, tal como argumentan Smith y Taylor (2010: 33), "las capacidades de los niños están fuertemente influidas por las expectativas y oportunidades de participación que les ofrece su cultura, así como por la cantidad de apoyo que reciben al adquirir nuevas competencias". Desde la perspectiva sociocultural, el niño se concibe como un agente activo, que construye activamente significados a partir de los relatos y narraciones en los que la cultura los va incorporando (Bruner, 1990). La educación, entonces, se entiende como un proceso de diálogo y transacción entre el adulto y el niño, donde ambos negocian y recrean el significado de la acción conjunta, y donde el niño tiene una voz protagónica, convirtiéndose en miembro de una comunidad creadora de cultura (Bruner, 1986). Siguiendo una argumentación similar, Wells (2001) propone que las aulas deben convertirse en "comunidades de indagación donde se considere que el currículo se crea de una manera emergente en los muchos modos de conversación con los que el enseñante y los estudiantes comprenden de una manera dialógica temas de interés individual y social por medio de la acción, la construcción de conocimiento y la reflexión” (p. 113). 
Estudios Pedagógicos XLI, $\mathrm{N}^{\circ}$ Especial: 81-96, 2015

ESCUCHANDO A LOS NIÑOS: SIGNIFICADOS SOBRE APRENDIZAJE Y PARTICIPACIÓN COMO EJES CENTRALES DE LOS PROCESOS DE INCLUSIÓN EDUCATIVA EN UN ESTUDIO EN ESCUELAS PÚBLICAS EN CHILE

Sin embargo, tal como se ha venido planteando en el debate sobre la relación entre las teorías del desarrollo y las prácticas sociales dirigidas hacia la infancia, lo que subyace a muchas de estas prácticas son determinadas teorías del desarrollo del niño que lo conciben como carente de aptitudes de comunicación, regulación y resolución de problemas (Bruner, 1986), así como también concepciones del aprendizaje entendido como transmisión de un conocimiento objetivo del profesor a los alumnos que estos deben recibir sin interrogar (Pozo et al., 2006). Estas concepciones no contribuyen al desarrollo de una educación respetuosa del derecho del niño a ser escuchado.

Conocer la perspectiva que los niños tienen de los procesos educativos, permitiría tener una visión práctica de qué aspectos cambiar y perfeccionar en la implementación de estrategias que promuevan la inclusión. Tal como señalan Rudduck y Flutter (2007, p. 40) "las ideas de su mundo pueden ayudarnos a ver cosas a las que normalmente no prestamos atención, pero [que] sí les importan”.

Tras lo planteado y con el objetivo de comprender las posibilidades y los límites de los procesos de reestructuración que supone la inclusión educativa, un elemento que resulta fundamental es la comprensión de los significados que los estudiantes atribuyen a sus experiencias cotidianas en la escuela, asignándole a estos un papel protagónico en la definición del diagnóstico de la situación, así como de las propuestas para su mejora. Al respecto, Fullan (2002) en el marco de la discusión sobre las reformas educativas advierte que pocas veces los adultos piensan en los estudiantes como participantes de la vida organizativa y de los procesos de cambio en la escuela, sino que simplemente los ven como beneficiarios de estos procesos. La conclusión de su análisis sobre el rol de los estudiantes en las iniciativas de cambio, mejora o innovación en educación es que "si no les asignamos algún papel significativo en la obra, la mayor parte del cambio educativo -y en realidad de la educación- fracasará" (p. 178).

En consecuencia, los objetivos del presente trabajo son describir los significados que los estudiantes construyen acerca del aprendizaje y la participación, con el propósito de aportar elementos para una evaluación crítica de políticas que buscan explícitamente promover la inclusión.

\section{MÉTODO}

Este estudio se desarrolló desde un diseño de investigación social cualitativo, con un enfoque narrativo. El encuadre desde la metodología cualitativa permite conocer significados que los participantes construyen sobre su propio contexto y comprender aspectos subjetivos y sociales sobre un fenómeno o un acontecimiento (Fernández, 2006; Flick, 2004). Asimismo, la metodología cualitativa es adecuada para "obtener detalles complejos de algunos fenómenos, tales como sentimientos, procesos de pensamiento y emociones" (Strauss \& Corbin, 2002: 21), lo que concuerda con el acercamiento a los significados de los estudiantes acerca de los procesos de aprendizaje y participación que en la presente investigación se quiere realizar.

El enfoque del estudio permite estudiar la experiencia por medio de la narrativa, indagando en la forma en que los humanos experimentan el mundo (Connelly \& Clandinin, 2008). White y Epston (1993) proponen que "para entender nuestras vidas y expresarnos a nosotros mismos, la experiencia debe 'relatarse', y que es precisamente el hecho de relatar 
lo que determina el significado que se atribuirá a la experiencia” (p. 27). Es decir que las personas van dando significado a su vida, en la medida en que convierten aquello que han vivido en relatos. De esta manera, son estos mismos relatos elaborados los que van configurando la forma de relacionarse con y en la vida.

\subsection{PARTICIPANTES}

Los estudiantes del estudio pertenecían a tres escuelas básicas y municipales de distintas comunas de la ciudad de Santiago. En particular, participaron estudiantes de quinto básico, cuyas edades se encontraban entre los 10 y 11 años. Cada curso presentaba una matrícula compuesta por 30 estudiantes aproximadamente. Todos los establecimientos educacionales contaban con proyectos de integración escolar (PIE) y los cursos con los que se trabajó contaban con estudiantes pertenecientes al PIE. Tanto los establecimientos escolares como los participante fueron definidos por medio de un criterio de accesibilidad (Valles, 1999). El primer paso en la relación con las instituciones fue informar a cada director o directora sobre los objetivos, actividades y consideraciones del estudio, resolviendo las dudas que surgieron en este diálogo. En un segundo momento, en una reunión con padres y apoderados se les explicó los objetivos del proyecto de investigación y en qué consistiría la participación de los estudiantes, tras lo cual la totalidad de estos aprobaron la participación de sus hijos mediante la firma de un formulario de consentimiento informado. En el caso de los estudiantes, en una visita al curso previa al inicio del taller se presentaron los objetivos y las actividades en las que participarían, explicando que la información que se produciría era confidencial y anónima, por lo cual sus nombres no iban a ser difundidos y recalcando que podían restarse en cualquier momento del proceso. Por otra parte, para resguardar la confidencialidad de los participantes, se modificaron sus nombres en las transcripciones y se difuminaron sus rostros en las fotografías que se mostraron como parte de la divulgación de los resultados.

\subsection{TÉCNICAS Y PROCEDIMIENTO PARA LA PRODUCCIÓN DE DATOS}

Para la producción de datos se realizó un taller grupal en el cual se utilizaron diversas técnicas que implicaban la participación activa de los sujetos. Tal como señalan Ibáñez y Michelazzo (2013), el uso del taller como herramienta permitió el despliegue de otras formas de expresión distintas a la palabra, posibilitando el acercamiento a dimensiones sensibles de las vivencias de los sujetos. En cada escuela se realizó un taller de cuatro sesiones, de aproximadamente 90 minutos cada una. Durante las sesiones se reunió a los estudiantes en grupos de cuatro o cinco participantes. La conformación inicial de los grupos se mantuvo en la realización del taller. Además, cada grupo estuvo coordinado por un facilitador, miembro del equipo de investigación. En la última sesión del taller en las escuelas se trabajó con todo el curso, disponiendo a los estudiantes en un semicírculo.

A lo largo del desarrollo de los talleres, se utilizaron distintas técnicas basadas en fotografía, dibujo, títeres y narración que tuvieron el objetivo de estimular la participación activa de los estudiantes. Transversalmente a las técnicas utilizadas, se ocupó la estrategia de grupo focal (Canales, 2006) como dispositivo para conocer los relatos de los participantes.

En la primera sesión se trabajó con fotoelicitación. Esta técnica permite que los participantes tomen un rol activo en la investigación, contextualizando y dando sentido 
a las imágenes presentadas (Arias, 2011). Se trata de una técnica que compromete a los participantes en la reflexión en torno a sus experiencias y permite que distintos sujetos con conocimientos, habilidades y perspectivas particulares, puedan dialogar acerca de la temática contenida en la imagen (Kaplan y Miles, 2005). En concreto, se les mostró a los estudiantes un conjunto de diez imágenes extraídas de internet. La selección de las imágenes se hizo luego de una discusión con el equipo de investigación y el principal criterio para su uso fue que representaran situaciones de diversidad y aprendizaje dentro del aula y de la escuela, con el fin de que los participantes pudieran relatar historias y experiencias relacionadas con estas temáticas.

En la segunda sesión se trabajó con fotografías tomadas por los mismos estudiantes. La fotografía participativa como técnica de investigación "busca que los involucrados asuman un rol activo en la reflexión y acción en torno a su realidad" (Valdivia, 2013, p. 6). En este caso, a los participantes se les entregó cámaras fotográficas con el propósito de que retratarán sus experiencias más significativas en cuanto a participación en la escuela, para posteriormente generar una discusión en torno a los relatos generados a partir de estas.

En la tercera sesión se utilizaron textos evocativos breves -elaboradas por el equipo de investigación para tal efecto- que relataban situaciones de aprendizaje cooperativo y de atención a la diversidad dentro de la escuela. Estas se leyeron a los estudiantes, con el fin de que ellos expresaran su opinión respecto a las situaciones mencionadas. También se pidió a los estudiantes que crearan soluciones a los conflictos o finales alternativos a los presentados en las historias. Los textos evocativos estimulan la imaginación, permitiendo a los participantes completar los vacíos del relato con significados personales, de este modo el investigador lo que hace es "contar una historia que permita a otros contar (se) la suya" (Hernández, 2008, p. 97). Posteriormente, se discutió sobre sus ideas para mejorar su escuela y sobre cómo sería una escuela ideal. Entonces se entregó a cada grupo una cartulina en blanco para que pudieran dibujar cómo sería para ellos una "escuela ideal", en la cual todos y todas pudieran aprender más. Cauduro et al. (2009) sostienen que el dibujo como técnica de investigación, por un lado, motiva la participación de los niños, y por otro, facilita la conexión entre abstracciones ideológicas y situaciones específicas, ya que en su realización los niños recurren a elementos personales y colectivos de la experiencia cultural.

En la cuarta y última sesión, a través de breves historias interpretadas por títeres, se interactuó con los participantes con el fin de que fueran interpelados sobre los temas planteados, en relación a situaciones de maltrato entre iguales, inclusión de estudiantes indígenas o con discapacidad, castigo injustificado por parte de un profesor, entre otras. Se optó por títeres, ya que su uso permite a los niños recrear experiencias vividas y enfrentarse a situaciones en un ambiente seguro (Helton \& Kotake, 2004, citado en Benzi, 2012). Al final de la sesión, se evaluó conjuntamente con los participantes el desarrollo de los talleres.

\subsection{PROCEDIMIENTO DE ANÁLISIS DE INFORMACIÓN}

Para el procesamiento de los datos se realizó un análisis de contenido en base a la propuesta que emerge desde la Teoría Fundamentada, considerando las etapas de la codificación abierta y la codificación axial (Strauss y Corbin, 2002). No obstante, nos parece oportuno explicitar que en la presente investigación no se contemplaba como objetivo la construcción de una teoría -como plantea la Teoría Fundamenta-, sino más bien indagar y conocer los 
significados construidos sobre un tema, de manera exploratoria (Hernández et al., 1991). Para realizar el análisis de los datos, se transcribió los audios de las entrevistas realizadas y se realizó una primera lectura y discusión de las entrevistas transcritas en el equipo de investigación. Luego, se procedió a efectuar el análisis por medio de la codificación abierta (Strauss y Corbin, 2002), en el que los códigos elaborados conservaron las palabras y expresiones de los participantes. A estos códigos se les denominó "códigos en vivo". En un segundo momento de codificación, se establecieron agrupamientos de códigos en vivo, los cuales fueron llamados "códigos conceptuales". Estos códigos conceptuales presentaron un nivel de abstracción mayor y más cercano al marco teórico del estudio, pero resguardando las palabras y expresiones utilizadas por los estudiantes. En la siguiente etapa, se desplegó un proceso de codificación axial (Strauss y Corbin, 2002) apoyado fundamentalmente por la creación de redes de relaciones, lo cual permitió ir estableciendo las subcategorías y categorías de análisis. Durante todo el proceso de codificación, y posterior categorización, se utilizó el programa computacional Atlas.ti.

\section{RESULTADOS}

Los resultados se presentan en torno a dos conceptos centrales que guiaron el análisis: aprendizaje y participación. Si bien se reconoce que la división es conceptual y en cierta medida arbitraria, pues ambos están íntimamente ligados en todo proceso educativo, se vuelve necesario ordenar así la información para facilitar el análisis y la lectura de los resultados.

\subsection{APRENDIZAJE}

En la Figura 1 los resultados están ordenados en torno a tres ejes: "nos gusta que el profesor nos explique", "aprendemos mejor entre compañeros" y "las clases son aburridas". Estos tres ejes corresponden a las categorías que surgieron del análisis. Además, se observan los códigos conceptuales que dan cuenta de los significados de aprendizaje que elaboran los estudiantes.

El primer eje denominado "nos gusta que el profesor nos explique" se asocia a la valoración que realizan los estudiantes de aquellos profesores que se demuestran cercanos y que favorecen la creación de un clima positivo en el aula. Asimismo, los estudiantes valoran que los docentes expliquen los contenidos de manera personalizada y a todos dentro del aula, sin hacer distinciones o mostrar preferencias por algunos de ellos. Por otra parte, según los relatos de los participantes, los castigos o el mal trato no contribuyen al aprendizaje. Estas ideas quedan reflejadas en la cita que se presenta a continuación:

las profesoras son simpáticas y nos dejan preguntarles o hacer cosas así como que no nos intimide tanto, porque algunas profesoras como la de matemáticas nos trata mal y no podemos reaccionar (...) (Tercera sesión). 
Figura 1. Significados, barreras y recursos para el aprendizaje

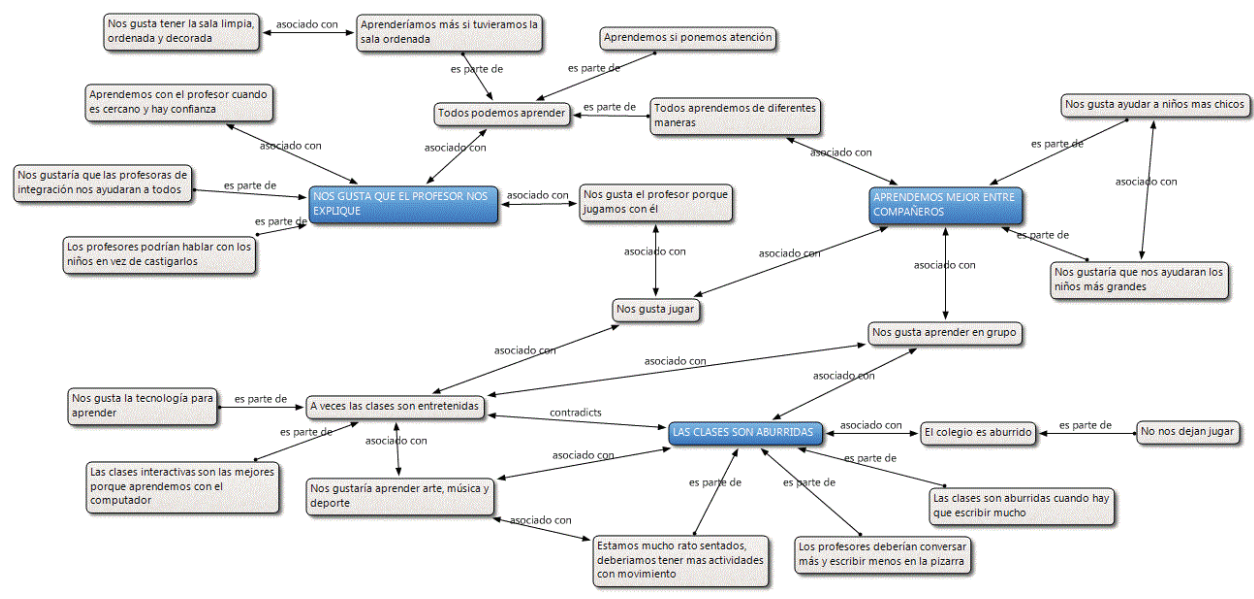

En el segundo eje "aprendemos mejor entre compañeros" se presentan elementos que relevan la importancia que tienen para los participantes las relaciones colaborativas entre ellos mismos y con pares de otros cursos. A continuación, se presenta un ejemplo:

¿Y qué beneficio tiene hacer una clase en grupo?

- Que nos ayudamos.

- Que nos ayudamos entre todos.

- Es mucho más fácil.

- Que si alguien tiene una duda, el otro lo puede ayudar, si no sabe el otro sí lo sabe.

- Y siempre termina el grupo completo.

- O con el diccionario, eh, yo busco tal palabra, ya, me salió esto y a la otra persona le salió otra cosa. Entonces como que las juntamos po’ (Primera sesión).

Así, diversos participantes afirman aprender más cuando los compañeros más competentes les explican los contenidos de ciertas asignaturas, sobre todo cuando los docentes solo dan explicaciones una vez. De esta manera, la interacción entre pares y su trabajo colaborativo, a pesar de no ser una práctica habitual, se constituye como recurso importante para el aprendizaje de los estudiantes. Asimismo, y aunque en los relatos se valora la eventual posibilidad de aprender de estudiantes mayores y de enseñar a estudiantes menores, los estudiantes manifiestan que no realizan actividades de este tipo.

En cuanto al tercer eje, sobre la metodología utilizada por los profesores en el aula, los estudiantes consideran que las clases son, en general, "aburridas". El tener que permanecer mucho tiempo sentados, escribir en exceso, que los contenidos se comuniquen en forma de "dictados" por parte de los profesores y que no se les permita jugar, son barreras que dificultan el aprendizaje de los estudiantes.

Chiquillos, y esas clases que ustedes dicen que son medias aburridas ¿qué se podría hacer para mejorarlas?

- Que hagan más clases divertidas. 
- Que griten menos, que no dicten muy rápido

- Que los profesores traten mejor a los niños

- Ah no, la otra clase que no me gusta...

- Y claro lenguaje...

- A mí tampoco me gusta lenguaje

- A veces me gusta lenguaje cuando hay que actuar o eso, pero cuando no hay que actuar, hacer una tarea en la pizarra, me aburro al tiro (Primera sesión).

En distintas ocasiones, los estudiantes señalan que ellos prefieren las clases en las que pueden jugar e interactuar con otros y aquellas en las que se utilizan recursos tecnológicos, tales como el computador, la pizarra interactiva o el proyector.

\subsection{PARTICIPACIÓN}

Al igual que en la red conceptual sobre aprendizaje, en la Figura 2 los significados se han organizado en torno a las categorías centrales de análisis, las que en el caso de la dimensión Participación son: "participo cuando juego y comparto con mis compañeros", "los profesores tienen estudiantes preferidos", "no me siento escuchado en la escuela" y "a los chicos no nos toman en cuenta en las decisiones"

Figura 2. Significados, barreras y recursos para la participación

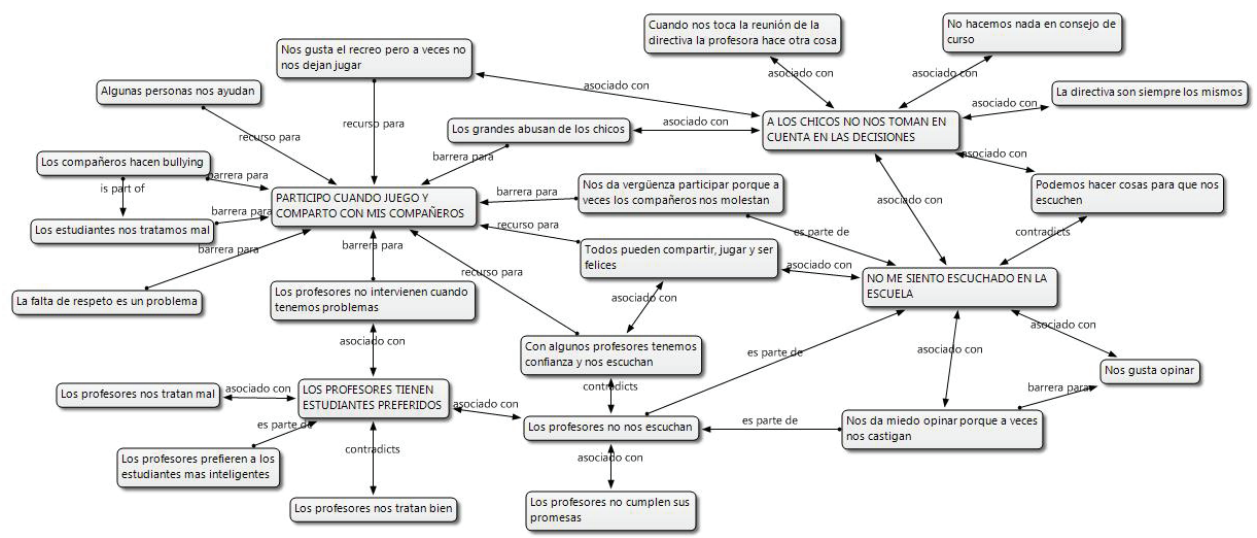

En cuanto al primer eje, para los estudiantes sentirse parte de su centro escolar depende, en gran medida, de poder compartir y jugar con libertad en compañía de sus compañeros, sentir el apoyo de los adultos en las cuestiones que les preocupan y no sentirse amenazados por los malos tratos con otras personas. Al igual como describimos en torno al aprendizaje, uno de los recursos presentes en la escuela se sostiene en relaciones de confianza y apoyo que los estudiantes tienen con sus pares y profesores. De esta manera, los participantes señalan que el recreo y las clases en que pueden trabajar en grupo son los momentos más agradables y esperados dentro de la jornada escolar. 
Ya, ¿y ustedes cuándo están felices en la escuela? ¿Cuándo se sienten felices?

- En la escuela, yo nunca.

¿Nunca? Ya, la Anita nunca, ¿y la Tamara?

- Cuando me hacen reír, cuando la Daniela, el Daniel, el Kevin hacen chistes (Segunda sesión).

Por otra parte, dentro de las narraciones desarrolladas, apreciamos que las burlas y las relaciones abusivas entre compañeros -particularmente desde estudiantes mayoresafectan de manera negativa la participación en la escuela.

¿Ustedes se sienten parte de la escuela o no?

- Yo no

¿Por qué no?

- Porque me molestan, no me escuchan, no me hablan, todos me tienen mala (Segunda sesión).

El segundo eje "los profesores tienen estudiantes preferidos", agrupa elementos discursivos referidos a las tensiones vividas en la relación con sus profesores donde lo que más se repite es la denuncia del favoritismo de algunos profesores hacia compañeros considerados como más inteligentes y de buen comportamiento. Asimismo, los estudiantes coinciden en que solo algunos profesores son considerados como "cercanos". La relación establecida con estos docentes está marcada por vínculos afectivos de confianza y por el interés hacia los estudiantes, mostrando disposición a conversar con ellos fuera del aula.

- Aquí hay dos, dos preferidos (...)

- Tres.

- La Rosa, la Carla y el Ángel (...)

- Porque la tía los quiere más a ellos

- Porque son... son inteligentes y la tía como que dice: 'no, es que acá hay niños que saben resolver súper bien en la pizarra'. Dice, así como mirando así, niños (...)

- Sí, como que uno está levantando la mano así de los primeros y después la levanta el Ángel, la

Rosa o la Carla y es como que, 'Ya Carla', 'Rosa', ‘Ángel' (Primera sesión).

Dentro del tercer eje, "no me siento escuchado en la escuela", se distingue la percepción que tienen los estudiantes de que no son considerados dentro de la escuela. Esta idea queda reflejada en la siguiente cita:

¿Por qué tú crees que no les preguntan nada?

- Porque somos niños chicos y no les importan las cosas que nosotros pensamos.

- Porque si importáramos nos deberían preguntar algo (Segunda sesión).

En el cuarto eje, "a los chicos no nos toman en cuenta en las decisiones", se observa a través de los relatos que las instancias que tienen los estudiantes para deliberar sobre algún aspecto de su educación son mínimas. Esto se constata en que los niños prácticamente perciben que no tienen las instancias formales ni las oportunidades por parte de los docentes de decidir sobre algún tema.

¿Por qué tú crees que no les preguntan nada?

- Porque somos niños chicos y no les importan las cosas que nosotros pensamos.

- Porque si importáramos nos deberían preguntar algo (Segunda sesión). 


\section{DISCUSIÓN}

El enfoque metodológico de producción y análisis de los datos del presente estudio, así como sus presupuestos teóricos, nos ha llevado a poner especial atención a las narraciones de niños y jóvenes. Los resultados obtenidos dan cuenta de una serie de significados que los estudiantes van construyendo sobre su experiencia escolar, significados relevantes para comprender en qué medida la escuela está fomentando el aprendizaje y la participación de todos y todas.

Para los participantes lo que cobra mayor importancia en los procesos de enseñanza y aprendizaje son las vivencias personales que ocurren en relaciones interpersonales socialmente significativas. En relación a los profesores, niños y jóvenes han expresado que los docentes deben ser capaces de explicar los contenidos a todos los estudiantes, que aprenden mejor cuando se da una relación de cercanía y confianza con el profesor y en las situaciones en que se utiliza el diálogo en lugar del castigo. En este sentido, resulta interesante considerar los hallazgos de Urbina et al. (2011) relativos a que aquellos docentes que privilegiaban la utilización de estrategias dialógicas para la resolución de la disrupción en el aula eran aquellos que percibían más apoyo de parte de la escuela para enfrentar dichas disrupciones.

Por otra parte, los estudiantes consideran que los "profesores de integración" deberían ayudar a todos los estudiantes. Reconocen, asimismo, la importancia de sus pares tanto en el ámbito socio-afectivo como académico, valorando instancias de juego y confidencialidad y de colaboración ante dudas que surgen en clases.

En otras palabras, los estudiantes abogan por un clima positivo de aprendizaje en el aula y reclaman un espacio en el que todos se sientan y sean reconocidos, donde todos puedan participar, dar su opinión y consultar sus dudas sin ser juzgados, discriminados o excluidos. Estas ideas refuerzan la creación de una buena convivencia escolar, favorecedora del aprendizaje.

Sin embargo, los niños y jóvenes del estudio han entregado una perspectiva crítica de lo que sucede en sus escuelas, siendo reiterativos en describir malos tratos por parte de los docentes y mencionar que en la escuela no los escuchan, que los "privilegios" son solo para algunos, que las instancias de participación no existen o son impuestas y que las situaciones de aprendizaje en aula son, en su mayoría, aburridas y en las cuales su participación es baja o nula. Esta situación es, a lo menos, compleja, si comprendemos que las experiencias de aprendizaje más valiosas y pertinentes son, frecuentemente, en las cuales se incita a los estudiantes a decidir y a participar (Wells, 2001).

Tanto la "escuela ideal" como las clases que los participantes proponen, se alejan de las tradicionales metodologías de enseñanza focalizadas en la trasmisión de conocimientos. Un ejemplo de esto es la práctica de aquellos profesores que copian o dictan contenidos respecto a un tema y que exige estudiantes silenciosos e inmóviles en sus sillas. Los niños son más lúdicos en su propuesta de enseñanza, planteando actividades donde puedan interactuar más con sus compañeros, con la tecnología, la música, las artes visuales, el deporte y el juego. En este sentido, las ideas de los estudiantes se acercan bastante a los planteamientos actuales respecto a la complejidad del aprendizaje que proponen que la mediación de significado no se limita al habla, es decir, que también otros modos de representación: dibujos, diagramas, mapas, danza, teatro, etc., se constituyen como medios de comunicación y, por lo tanto, en instrumentos con los que pensar y aprender (Wells, 
2001). Los datos producidos pueden interpretarse, de manera similar, a la luz de los principios del diseño universal de aprendizaje (CAST, 2008).

Por lo demás, el artículo 31 de la Convención sobre los Derechos del Niño declara de manera explícita que "los Estados partes reconocen el derecho del niño al descanso y el esparcimiento, al juegoy alas actividades recreativas propias de su edad y a participar libremente en la vida cultural y en las artes" (p. 5). Más específicamente, el Comité de los Derechos del Niño [CDN] (2013) estipula que "la educación y el juego incluyentes se refuerzan entre sí y deben facilitarse cotidianamente en la educación y los cuidados de la primera infancia (preescolares), así como en la escuela primaria y secundaria" (p. 10). Y, aunque parece haber un acuerdo entre los estudiantes de quinto año básico en que el juego resulta una característica importante para las experiencias de aprendizaje, este no es uno de los elementos que se consideran relevantes para el diseño de la enseñanza en sus escuelas. Al respecto, el Comité de los Derechos del Niño reconoce que el juego y la recreación son vitales para todos los aspectos del aprendizaje, la promoción del bienestar, la salud y el desarrollo de la imaginación, la creatividad y aptitudes físicas, emocionales, cognitivas y sociales, y manifiesta su preocupación por el escaso reconocimiento que se otorga a estos derechos. Asimismo, el juego y la recreación tienen un valor intrínseco por el placer y disfrute que generan, contribuyendo a que los niños exploren y aprendan en la práctica, favoreciendo capacidades de negociación, toma de decisiones, resolución de conflictos e innovación (CDN, 2013).

En este punto, la voz de estos estudiantes es coherente con los argumentos de la ciencia contemporánea. Tal como argumentan Whitebread et al. (2012), el juego ha permitido la evolución de un ser humano con mayor capacidad de aprender y resolver problemas, favoreciendo el desarrollo sináptico de la corteza frontal y el desarrollo de habilidades de representación simbólica y de autorregulación. Por lo demás, la ausencia de este tipo de actividades aumentaría el riesgo de estrés y problemas de salud mental infantil. Asimismo, el juego se constituye en una herramienta que puede proporcionar a los docentes una mejor comprensión y comunicación con los estudiantes, además de entregar oportunidades para el intercambio intergeneracional (CDN, 2013) y, por lo tanto, para la negociación y acuerdo de significados comunes (Bruner, 1986).

En este sentido, y en consideración de las demandas de los estudiantes, cabe formular la necesidad de una figura adulta, en este caso el docente, que apoye la construcción de un contexto de diálogo conjunto, en el que todos puedan "sentirse parte" (CDN, 2013). Igualmente, la sensibilidad de los profesores ante la incertidumbre de las actividades de aprendizaje, así como la propuesta de tareas que faciliten la participación, favorecen el autoconcepto positivo de los estudiantes (Geddes, 2006).

En esta misma línea, cabe mencionar que hoy existe un amplio acuerdo (Bruner, 1986) en que un mecanismo fundamental del desarrollo es la interacción del niño con pares y adultos más competentes mediante la realización de actividades conjuntas de cooperación. Al respecto, Vygotski (1978) fue uno de los primeros que, hace ya varias décadas, argumentó convincentemente la importancia de las relaciones interpersonales y la resolución conjunta de problemas en el desarrollo, cuando afirmaba que: "todas las funciones superiores se originan como relaciones entre seres humanos" (p. 94). De igual modo, pareciera que las necesidades expresadas por los estudiantes apuntan a una práctica pedagógica coherente con los principios del andamiaje y el ajuste de la ayuda pedagógica, esto es, el apoyo eficaz que el adulto proporciona ajustándose de forma dinámica a las competencias y necesidades del niño en cada momento (Onrubia, 2009). 
Por otro lado, suele suceder que el profesorado - presionado por múltiples factores- se "obsesione" con el contenido y el rendimiento de los estudiantes, inhibiendo su capacidad de respuesta para prestar atención a la experiencia emocional del aprendizaje. Y, cuando en nuestro sistema educativo hay un énfasis importante en que el éxito académico sea el único exponente del éxito educativo, puede resultar muy perjudicial para el desarrollo, expectativas, bienestar y autoestima de los estudiantes (Geddes, 2006). Cuando los estudiantes expresan que los profesores los tratan de manera injusta, que los niños más grandes cometen abusos hacia los más chicos, que los profesores no intervienen ante conflictos o peleas entre compañeros, constatamos elementos que no favorecen un ambiente positivo de aprendizaje.

En este sentido, Aron y Milicic (1999) afirman que las consecuencias del clima escolar repercuten en todos los procesos de la comunidad escolar, incluyendo, entre otros, la calidad de los vínculos establecidos y el desarrollo y aprendizaje de los estudiantes. De la misma manera, Durán (2009) explicita la importancia de la instauración de un ambiente colaborativo y de ayuda mutua entre pares, pues además crecer en una cultura de colaboración y participación va propiciando que los niños desarrollen estas respuestas no solo en la sala de clases, sino que también en otros contextos.

Hasta aquí hemos descrito que los estudiantes aprenden en un contexto adverso a la diversidad, donde se plantean contenidos y metodologías poco pertinentes a sus intereses y habilidades, en una cultura de aprendizaje que, además, no aprovecha el recurso de colaboración y la solidaridad. Al respecto, la perspectiva de la inclusión educativa plantea una reestructuración radical de las formas tradicionales de entender la enseñanza y el aprendizaje, que enfatiza la participación de los estudiantes reconociendo su derecho a ser escuchados y tomar parte de las decisiones referidas a su educación, de modo que progresivamente se conviertan en agentes causales de su existencia (Wehmeyer, 2009). Esta manera de comprender el proceso educativo coincide con las aproximaciones socioculturales del desarrollo desde las cuales se concibe al niño como un ser en transformación que construye activamente significados de su experiencia y cuyas capacidades dependen de las oportunidades de participación y del apoyo que les ofrecen adultos y pares en su contexto (Smith \& Taylor, 2010).

Sin embargo, las prácticas escolares tradicionales no han puesto énfasis en promover la participación activa de los niños en la resolución de los problemas que les afectan, ni en los significados que construyen sobre su aprendizaje, ni tampoco en su autodeterminación. Beane y Apple (2000) han sostenido que "después de todo, a pesar de la retórica de la democracia en nuestra sociedad y la idea de sentido común de que la forma de vida democrática se aprende a través de las experiencias democráticas, las escuelas han sido instituciones notablemente poco democráticas" (p. 28). Rudduck y Flutter (2007), por su parte, argumentan que "la presunción cómoda más duradera, que ha configurado la política y la práctica en muchos aspectos de la vida, ha sido que la infancia está relacionada con la dependencia" (p. 19) y que en las escuelas se sigue creyendo que "la mayoría de los jóvenes carece aún de la capacidad de influir en la calidad de su vida" (p. 20). Ello explica que no haya un interés significativo por las experiencias de los estudiantes ni que se suelan ofrecer oportunidades para que estos expresen su malestar (o bienestar) o sus sensaciones y pensamientos de lo que les pasa en su contexto educativo (Rudduck \& Flutter, 2007).

Recapitulando, los estudiantes se sienten parte de la escuela en la medida que los profesores y compañeros los tratan con respeto, sienten apoyo y amistad con otros 
miembros de la comunidad escolar y perciben un clima en que pueden compartir, jugar y ser felices. De igual forma, se sienten escuchados cuando no se sienten avergonzados por los comentarios que realizan o cuando los profesores no los castigan por decir lo que piensan. La participación es fundamental en la medida que favorece el sentido de pertenencia de los miembros, resultando además necesaria para poder comprender tanto la propia cultura como la de otros, ampliando sus horizontes y contribuyendo a la empatía mutua y a la valoración de la diversidad (CDN, 2013).

A diferencia de lo que podría esperarse de un trabajo situado en la perspectiva de la inclusión educativa, en este estudio no hemos focalizado nuestra atención en estudiantes con discapacidad o identificados con alguna otra categoría. La intención ha sido, partiendo desde una perspectiva de derechos, explorar significados de los estudiantes como grupo, enfatizando lo que tienen en común antes que sus diferencias y en consideración de que niños y adolescentes tienen derecho a expresar sus opiniones en todos los asuntos que los afectan. Asimismo, nos llama la atención la sensibilidad manifestada por los estudiantes hacia las situaciones de injusticia, lo cual nos lleva a mirar los problemas de la inclusión no solo desde la perspectiva de las dificultades que pueden tener los niños para aprender, sino también a abordarlos desde un marco mucho más amplio que pone el acento sobre el desarrollo de comunidades escolares acogedoras y respetuosas de todos y todas. Booth y Ainscow (2000) han sostenido que la inclusión educativa consiste básicamente en poner determinados valores en acción, y los referidos a igualdad y justicia resultan fundamentales en este marco.

Con todo, creemos que el trabajo de investigación aquí presentado aporta perspectivas que enriquecen el debate sobre inclusión educativa, escuchando lo que los estudiantes dicen respecto a lo que esperan y proponen sobre su proceso educativo y su espacio escolar. Las ideas que surgen de este análisis enfatizan aspectos a nuestro juicio esenciales sobre los que incidir para la "reestructuración" necesaria de las prácticas pedagógicas, así como para la transformación de las bases de la convivencia entre profesores y estudiantes y de la comunidad escolar en su conjunto. Ciertamente, creemos que esta discusión forma parte del campo de conocimiento que identificamos como inclusión educativa, en el entendido que parte importante de la tarea consiste en crear comunidades escolares acogedoras en las que todos se sientan parte y sientan que aprenden y desarrollan su potencial para colaborar con otros en proyectos comunes. Finalmente, la discusión sobre cómo atender a la diversidad requiere ampliar la comprensión respecto no sólo de lo que nos diferencia, sino también respecto a aquello que como seres humanos compartimos y nos hace semejantes.

\section{REFERENCIAS BIBIOGRÁFICAS}

Ainscow, M., \& Miles, S. (2009). Desarrollando sistemas de educación inclusiva: ¿Cómo podemos hacer progresar las políticas? En C. Giné (Coord.), La educación inclusiva. De la exclusión a la plena participación de todo el alumnado (pp. 161-170). Barcelona: Horsori Editorial.

Ainscow, M., Booth, T., \& Dyson, A. (2006). Improving schools, developing inclusion. Londres: Routledge.

Arias, D. (2011). El co-relato de la imagen fotográfica: la arqueología visual como metodología en la exploración de la memoria etnohistórica. Quaderns-e de l'Institut Català d'Antropologia, 16(1-2), 173-188. Recuperado desde http://www.antropologia.cat/quaderns-e-178

Aron, A., \& Milicic, N. (1999). Clima social escolar y desarrollo personal: Un programa de 
Estudios Pedagógicos XLI, $\mathrm{N}^{\circ}$ Especial: 81-96, 2015

ESCUCHANDO A LOS NIÑOS: SIGNIFICADOS SOBRE APRENDIZAJE Y PARTICIPACIÓN COMO EJES CENTRALES DE LOS PROCESOS DE INCLUSIÓN EDUCATIVA EN UN ESTUDIO EN ESCUELAS PÚBLICAS EN CHILE

mejoramiento. Santiago: Andrés Bello.

Beane, J., \& Apple, M. (2000). La defensa de las escuelas democráticas. En J. Beane y M. Apple (Coords.), Escuelas Democráticas (pp.13-47). Madrid: Ediciones Morata.

Benzi, G. (2012). Títeres en psicoterapia constructivista. En M. Sepúlveda, C. Alcaíno y G. Alonso (Eds.), Psicoterapia evolutiva constructivista en niños y adolescentes. Métodos y técnicas terapéuticas. Juegos, imágenes, diálogos (pp. 69-79). Santiago: Universidad de Chile.

Black-Hawkins, K., Florian, L., \& Rouse, M. (2007). Achievement and inclusion in schools. Londres: Routledge.

Blanco, R. (2006). La equidad y la inclusión social: uno de los desafíos de la educación y la escuela de hoy. Revista Electrónica Iberoamericana sobre Calidad, Eficacia y Cambio en Educación, 4(3), 1-15.

Booth, T., \& Ainscow, M. (2000). Índice de inclusión: desarrollando el aprendizaje y la participación en las escuelas. Bristol: Centre for Studies on Inclusive Education (CSIE).

Bruner, J. (1986). Realidad mental y mundos posibles. Barcelona: Gedisa.

Bruner, J. (1990). Actos de significado. Más allá de la revolución cognitiva. Madrid: Alianza Editorial.

Canales, M. (2006). Metodologías de la investigación social. Santiago: LOM.

CAST (2008). Universal design for learning guidelines version 1.0. Wakefield, MA.

Cauduro, M., Birk, M., \& Wachs, P. (2009). Investigación basada en las artes: una aportación brasileña. Forum: Qualitative Social Research, 10(2), 1-18. Recuperado desde http://nbn-resolving. de/urn:nbn:de:0114-fqs0902335

Comité de los Derechos del Niño (2013). Observación general $N^{o} 17$ (2013) sobre el derecho del niño al descanso, el esparcimiento, el juego, las actividades recreativas, la vida cultural y las artes (artículo 31). Ginebra: Organización de Naciones Unidas.

Connelly, F.M., \& Clandinin, D.J. (2008). Relatos de experiencia e investigación narrativa. En J. Larrosa, R. Arnaus, V. Ferrera, N. Pérez de Lara, F.M. Connelly, D.J. Clandinin y M. Greene (Eds.), Déjame que te cuente. Ensayos sobre narrativa y educación (pp. 11-59). Buenos Aires: Laertes.

Durán, D. (2009). El aprendizaje entre alumnos como apoyo a la inclusión. En C. Giné (Coord.), La educación inclusiva. De la exclusión a la plena participación de todo el alumnado. Barcelona: Horsori.

Fernández, R. (2006). Investigación cualitativa y psicología social crítica en el Chile actual: Conocimientos situados y acción política. Forum Qualitative Social Research, 7(4). Recuperado desde http://www.qualitative-research.net/index.php/fqs/article/view/163/364

Flick, U. (2004). Introducción a la Investigación Cualitativa. Madrid: Ediciones Morata.

Fullan, M. (2002). Los nuevos significados del cambio en educación. Barcelona: Editorial Octaedro.

Geddes, H. (2010). El apego en el aula. Relación entre las primeras experiencias infantiles, el bienestar emocional y el rendimiento escolar. Barcelona: Editorial Graó.

Hernández, F. (2008). La investigación basada en las artes. Propuestas para repensar la investigación en educación. Educatio Siglo XXI, 26, 85-118. Recuperado desde http://revistas.um.es/ educatio/article/view/46641/44671

Hernández, R., Fernández, C. y Baptista, P. (1991). Metodología de la Investigación. México: McGrawHill.

Ibáñez, I., \& Michelazzo, C. (2013). Expresividades escópico, espacialidad y sensibilidades. Forum Qualitative Social Research, 14(1). Recuperado de http://www.qualitative-research.net/ index.php/fqs/article/view/1896/3505

Infante, M. (2007, Septiembre). Inclusión educativa en el cono sur: Chile. Taller Regional Preparatorio sobre Educación Inclusiva América Latina, Regiones Andina y Cono Sur, UNESCO, Buenos Aires.

Kaplan, I., \& Miles, S. (2005). Using images to promote reflection: an action research study in 
Estudios Pedagógicos XLI, Nº Especial: 81-96, 2015

ESCUCHANDO A LOS NIÑOS: SIGNIFICADOS SOBRE APRENDIZAJE Y PARTICIPACIÓN COMO EJES CENTRALES

DE LOS PROCESOS DE INCLUSIÓN EDUCATIVA EN UN ESTUDIO EN ESCUELAS PÚBLICAS EN CHILE

Zambia and Tanzania. Journal of Research in Special Educational Needs, 5(2), 77-83.

Lansdown, G. (2005). The evolving capacities of the child. Florencia: UNICEF.

López, V., Julio, C., Pérez, M.V., Morales, M., \& Rojas, C. (2014). Barreras culturales para la inclusión: políticas y prácticas de integración en Chile. Revista de Educación, 363(1), 256-281.

Onrubia, J. (2009) Transformar para adaptar, adaptar para incluir: una mirada psicoeducativa a la educación inclusiva. En C. Giné (Coord.), La educación inclusiva. De la exclusión a la plena participación de todo el alumnado (pp. 49-62). Barcelona: Horsori.

Parrilla, A. (2009). ¿Y si la investigación sobre inclusión no fuera inclusiva? Reflexiones desde una investigación biográfica-narrativa. Revista de Educación, 349, 101-118.

Pozo, J. I., Scheuer, N., Pérez Echeverría, M. P, Mateos, M., Martín, E., \& de la Cruz, M. (Eds.) (2006). Nuevas formas de pensar la enseñanza y el aprendizaje. Las concepciones de profesores y alumnos. Barcelona: Graó.

Rogoff, B. (1997). Los tres planos de la actividad sociocultural: "Apropiación Participativa", "Participación Guiada” y “Aprendizaje”. En J. Wertsch, P. del Río y A. Álvarez (Comps.), La mente sociocultural. Aproximaciones teóricas y aplicadas (pp. 111-128). Madrid: Fundación Infancia y Aprendizaje.

Rudduck, J., \& Flutter, J. (2007). Cómo mejorar tu centro escolar dando la voz al alumnado. Madrid: Ediciones Morata.

Slee, R. (2012). La escuela extraordinaria. Exclusión, escolarización y educación inclusiva. Madrid: Ediciones Morata.

Smith, A. B., \& Taylor, N. (2010) El contexto sociocultural de la infancia: el equilibrio entre dependencia y autonomía. En A. B. Smith, N. J. Taylor y M. M. Gollop (Eds.), Escuchemos a los niños. México: Fondo de Cultura Económica.

Strauss, A., \& Corbin, J. (2002). Bases de la Investigación Cualitativa. Medellín: Editorial de la Universidad de Antioquia.

United Nations Educational, Scientific and Cultural Organization [UNESCO]. (2008). Educación Inclusiva: el Camino hacia el Futuro. Conferencia Internacional sobre Educación. 48va reunión. Ginebra: Autor.

Urbina, C. Simón, C., \& Echeita, G. (2011). Concepciones de los profesores acerca de las conductas disruptivas: análisis a partir de un marco inclusivo. Infancia y Aprendizaje 34(2), 205-217.

Valdivia, C. (2013). La imagen es tu voz: la fotografía participativa como herramienta de cambio social. Revista Canalé, 6(5), 6-16. Recuperado de http://facultad.pucp.edu.pe/comunicaciones/ files/2014/03/Canale_05_2013.pdf

Valles, M. (1999). Técnicas cualitativas de investigación social. Reflexión metodológica y práctica profesional. Madrid: Editorial Síntesis.

Vygotski, L. (1978). Mind in society. The development of higher psychological processes. Cambridge, MA: Harvard University Press.

Wehmeyer, M. (2009). Autodeterminación y tercera generación de prácticas de inclusión. Revista de Educación, 349, 45-67.

Wells, G. (2001). Indagación dialógica: hacia una teoría y una práctica socioculturales de la educación. Barcelona: Paidós.

White, M., \& Epston, D. (1993). Medios narrativos para fines terapéuticos. Barcelona: Editorial Paidós.

Whitebread, D., Basilio, M., Kuvalja, M., \& Verma, M. (2012). The importance of play: a report on the value of children's play with a series of policy recommendations. Bruselas: Toys Industries for Europe. 